\title{
Cost-Efficient Mobile Crowdsensing with Spatial-Temporal Awareness
}

\author{
Qin Hu, Shengling Wang, Member, IEEE, Xiuzhen Cheng, Fellow, IEEE, \\ Junshan Zhang, Fellow, IEEE, and Weifeng Lv
}

\begin{abstract}
A cost-efficient deal that can achieve high sensing quality with a low reward is the permanent goal of the requestor in mobile crowdsensing, which heavily depends on the quantity and quality of the workers. However, the spatial diversity and temporal dynamics lead to heterogeneous worker supplies, making it hard for the requestor to utilize a homogeneous pricing strategy to realize a cost-efficient deal from a systematic point of view. Therefore, a cost-efficient deal calls for a cost-efficient pricing strategy, boosting the whole sensing quality with less operation (computation) cost. However, the state-of-the-art studies ignore the dual cost-efficient demands of large-scale sensing tasks. Hence, we propose a combinatorial pinning zero-determinant (ZD) strategy, which empowers the requestor to utilize a single strategy within its feasible range to minimize the total expected utilities of the workers throughout all sensing regions for each time interval, without being affected by the strategies of the workers. Through turning the worker-customized strategy to an interval-customized one, the proposed combinatorial pinning ZD strategy reduces the number of pricing strategies required by the requestor from $O\left(n^{3}\right)$ to $O(n)$. Besides, it extends the application scenarios of the classical ZD strategy from two-player simultaneous-move games to multiple-heterogeneous-player sequential-move ones, where a leader can determine the linear relationship of the players' expected utilities. Such an extension enriches the theoretical hierarchy of ZD strategies, broadening their application scope. Extensive simulations based on real-world data verify the effectiveness and efficiency of the proposed scheme.
\end{abstract}

Index Terms-Mobile crowdsensing, pricing strategy, quality control, game theory.

\section{INTRODUCTION}

$\mathrm{M}$ OBILE crowdsensing (MCS), essentially a practice of sharing economy, leverages the Internet to elicit scattered sensing services from a large group of participants for accomplishing a complex task [1]-[4]. As Adam Smith mentioned in The Wealth of Nations, the market capacity limits the division. MCS expands the capacity of the originally scattered market by the Internet, making it large enough to facilitate a refined task division. As a result, MCS is capable of breaking through a variety of market limitations and barriers, exploiting and sharing the offthe-shelf sensing resources economically and efficiently. The merits of MCS contribute to its wide study in various fields, such as environmental quality assessment [5], transportation monitoring [6], and outdoor positioning [7]. Commercial MCS sites and applications (e.g., Field Agent [8], Gigwalk [9], Waze [10], and Million Agents [11]) also go into services.

In a typical MCS, the requestor (crowdsourcer) recruits

- Qin Hu is with the Department of Computer and Information Science, Indiana University - Purdue University Indianapolis, IN, USA.

E-mail: qinhu@iu.edu

- Shengling Wang (corresponding author) is with the College of Information Science and Technology, Beijing Normal University, Beijing, China. E-mail: wangshengling@bnu.edu.cn

- Xiuzhen Cheng is with the Department of Computer Science, The George Washington University, Washington DC, USA.

E-mail:cheng@gwu.edu

- Junshan Zhang is with the School of Electrical, Computer and Energy Engineering, Arizona State University, Tempe, AZ, USA.

E-mail: junshan.zhang@asu.edu

- Weifeng $L v$ is with the School of Computer Science and Engineering, Beihang University, Beijing, China.

E-mail: lwf@buaa.edu.cn workers (crowdsourcees) to cooperatively perform a complicated sensing job by rewarding with economic profits. A cost-efficient deal that can bring high sensing quality with a low reward is the basic value and permanent goal of the requestor, which heavily depends on the quantity and quality of the workers. However, the spatial diversity and temporal dynamics lead to heterogeneous worker supplies. For example, as shown in Fig. 1, the heat maps of the check-in activities during 6:00-7:00 and 12:0013:00 in New York City from Foursquare [12] illustrate the dramatically distinct numbers of visitors in different areas and time intervals. On one hand, when opportunistic sensing is adopted, such visitors are potential worker candidates, which are obviously uneven in both spatial and temporal dimensions; on the other hand, if participatory sensing is employed, the workers may have their own preferences on when and where to provide sensing services. That is, for both mainstream sensing modes, the differences in space and time give rise to divergent worker candidate pools.

The heterogeneous worker supplies make it difficult for the requestor to utilize a homogeneous pricing strategy for achieving a cost-efficient deal from a systematic viewpoint. Meanwhile, it is challenging to lay down fine-grained pricing strategies with spatial-temporal awareness for the requestor, especially for large-scale sensing tasks that involve a wide area with a long time span such as the longterm urban pollution monitoring. This is because it is a long haul for the requestor to design optimal pricing strategies for all workers in different intervals at target sensing regions in a one-by-one mode. Hence, a cost-efficient deal calls for a cost-efficient pricing strategy, boosting the whole sensing 


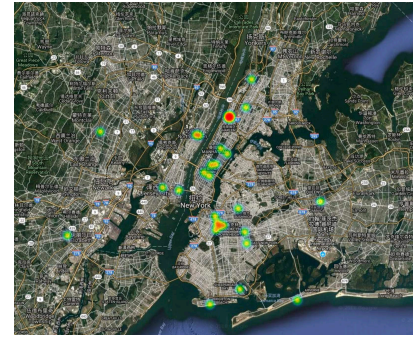

(a) 6:00-7:00

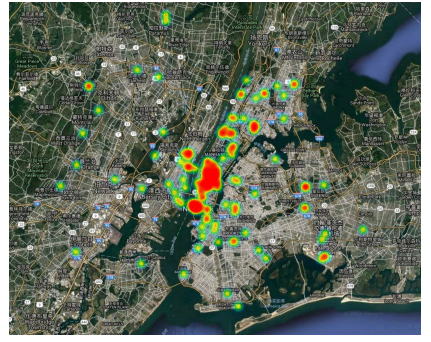

(b) 12:00-13:00
Fig. 1. Heat maps for check-in venues in New York City on Apr. 4, 2012.

quality with less computational cost.

However, the state-of-the-art studies, e.g., workselection-based [13]-[15], task-allocation-based [16]-[18] and incentive-based methods [19]-[21], ignore the dual costefficient demands, i.e., realizing a cost-efficient deal with a cost-efficient strategy, of large-scale sensing tasks. In fact, it is challenging to fulfill such demands since blindly reducing the payment to a worker is harmful to the utility of the requestor due to the reduced sensing quality, which is a natural cascading effect exerted by the worker. To address this challenge, an intuitive idea is to seek equilibrium strategies of the requestor and the worker in their game, under the assumption stating that all players are equal. Thus, the optimal utility of the requestor is finally achieved under the bridles of the workers.

In this paper, we tackle the above challenge from a different angle, by answering the following greedy question: is it possible for the requestor to minimize the utility of the worker while maximizing that of herself ${ }^{1}$ without being vigilant about the actions a worker may take? The answer is yes when we resort to the idea of the zero-determinant (ZD) strategy [22]. The classical ZD strategy provides us with a revolutionary understanding of Markovian games, with which the adopter (the ZD player) can unilaterally set the expected utility of the opponent no matter how the opponent acts. With the idea of $\mathrm{ZD}$, the requestor is not affected by the actions of the worker, dominating the game to pursue a cost-efficient deal.

Nevertheless, it is costly to directly apply the ZD strategy for meeting the dual cost-efficient demands in that it is essentially a kind of worker-customized strategy, making the number of the requestor's strategies to be $n_{a} \times b_{i} \times n_{t}$, where $n_{a}$ and $n_{t}$ are respectively the total numbers of target sensing regions and sensing intervals, and $b_{i}$ denotes the number of workers in sensing region $i \in\left\{1, \cdots, n_{a}\right\}$. To lay down a cost-efficient strategy, we propose a combinatorial pinning $Z D$ strategy, which empowers the requestor to utilize a single strategy within its feasible range to minimize the total expected utilities of the workers throughout all sensing regions for each interval, without being affected by the strategies of the workers. Through turning the workercustomized strategy to an interval-customized one, our combinatorial pinning ZD strategy reduces the number of pricing strategies required by the requestor to $n_{t}$. As a result, cost-efficient MCS with spatial-temporal awareness

1. For better differentiation, we regard the worker as "he" and the requestor as "she". is achieved, whose effectiveness and efficiency are verified based on real-world data.

The proposed combinatorial pinning ZD strategy has the following merits beyond contributing to meeting the dual cost-efficient demands:

1) it extends the application domain of the classical ZD strategy from two-player simultaneous-move games to multiple-heterogeneous-player sequentialmove games;

2) it empowers a leader to solely determine the linear relationship of the expected utilities of itself and all the followers in a sequential game, based on which a special case can be easily derived where the leader can set a fixed value to the weighted sum of the heterogeneous followers' expected utilities, no matter how the followers react;

3) it enriches the theoretical hierarchy of ZD strategies, broadening their application scope.

The rest of the paper is organized as follows. We introduce the most related work in Section 2. The problem formulation is presented in Section 3. Section 4 displays the derivation of the combinatorial pinning ZD strategy, based on which the detailed solution for the cost-efficient MCS is proposed in Section 5. Experimental evaluation is reported in Section 6 and the whole paper is concluded in Section 7.

\section{Related Work}

Most MCS research focuses on sensing quality and pricing, the two key elements of MCS. Quality control studies can be classified into three categories: worker-selection-based, task-allocation-based, and incentive-mechanism-based. The first category controls sensing quality by selecting qualified workers [13]-[15]. For examples, Song et al. [13] proposed a dynamic selection mechanism to choose the most appropriate set of participants so as to meet the quality of information constraint with the consideration of workers' various properties such as sensing ability, mobility, and so on; while Zhang et al. [14] employed a concept of coverage quality to evaluate the quality of the recruited workers, based on which they presented an approximation algorithm to select a proper worker subset; and in [15], a framework was put forward to match qualified workers with sensing tasks based on the measurement of each worker's coverage potential.

In task-allocation-based quality control, the best overall sensing quality is achieved through optimally assigning subtasks to selected workers [16]-[18]. In [16], Estrada et al. presented a time-constrained task allocation framework where a quality-aware task allocation algorithm was implemented based on Particle Swarm Optimization. In [17], the sensing quality of each task was taken into consideration and task-specific minimal sensing quality thresholds were introduced to redefine the multi-task allocation problem, based on which a novel framework named MTakser was proposed using a descent greedy approach. Wang et al. [18] focused on the overall sensed data error throughout the sensing areas instead of the traditional sensing coverage ratio based quality metric, and proposed a task allocation 
framework to dynamically select sensing subareas for reducing the number of task assignments.

Incentive-mechanism-based schemes integrate quality control into incentive mechanisms for MCS [19]-[21]. In [19], a quality-driven incentive mechanism based on the reverse Vickrey auction was proposed to maximize the social welfare, where each worker was paid according to the submitted quality. Similarly, Jin et al. [20] designed two incentive mechanisms incorporating the metric of workers' quality of information, which relied on reverse combinatorial auctions, including both the single-minded and multi-minded models. Besides, Peng et al. [21] adopted the expectation maximization algorithm and Bayesian inference to predict the data quality, and further employed an information theory based model to measure the effectiveness of the contributed data.

Pricing is another means to realize quality control in MCS [23], [24]. In [23], Han et al. studied the pricing problem with the consideration of sensing quality in MCS and solved this NP-hard problem using the properties of Poisson binomial distributions. Tian et al. [24] proposed an online learning technique based pricing scheme after revealing the data quality assessment principle in the MCS-based wireless fingerprint scenarios. Note that none of the works mentioned above considered the dual costefficient demands in MCS or the challenges resulted from the spatiotemporal heterogeneity of the worker supplies to design a cost-efficient pricing strategy for the requestor so as to realize a cost-efficient deal, which stimulates our work in this paper.

To be specific, we utilize the multi-player sequential game to model the interactions between the requestor and the workers in an MCS and develop the combinatorial pinning ZD strategy for this game, which is exactly an extension of the original ZD strategy. Generally speaking, existing extensions of the ZD strategy are mainly carried out from two perspectives: the number of players and the order of actions. For the former, Pan et al. [25] first explored the extension of the ZD strategy for the iterated public goods game involving multiple players with simultaneous moves, and Hilbe et al. [26] further proposed the concept of ZD alliance, and then continued to analyze the evolutionary performance of the ZD strategy in the multi-player game [27]; while for the latter, $\mathrm{Hu}$ et al. [28] exerted their efforts in studying the sequential ZD strategy for the twoplayer sequential game and applying it to enhance the crowdsourcing quality.

\section{Problem Formulation}

A large-scale mobile crowdsensing scenario is considered in this paper, where a long-term sensing task needs to be completed by workers located at different regions. The requestor is allowed to offer each worker a normal payment or an underpayment and she can employ the same worker for many times. Hence, after a worker witnesses the payment of the requestor, the inherent profitdriven property and the willingness of providing long-term services drive him to decide whether or not to submit highquality sensing data. The higher the submission quality, the higher the sensing cost and the higher the job opportunity in the future, and vice versa. The working process in one round of MCS is illustrated in Fig. 2.

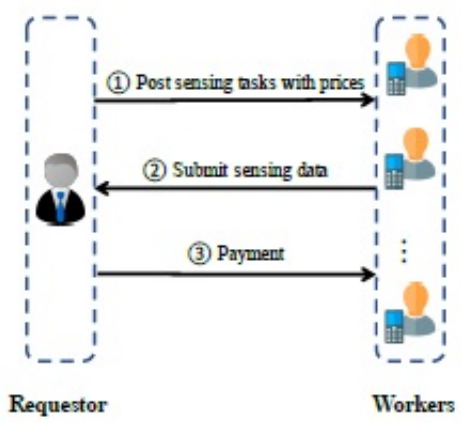

Fig. 2. Working process in one round of MCS.

The features of the repeated interactions, sequential decision-making, and multi-participation are highly compatible with the characteristics of the iterated multiplayer sequential game. Therefore, we take advantage of this game to model the interplay between the requestor and the workers. Specifically, each player in this game has two actions: cooperation, denoted as $c$, and defection, indicated by $d$. The cooperation action of the requestor is to offer the normal payment while her defection action is underpayment; for any worker in a certain sensing region, with the awareness of the requestor's action, he can choose the action $c$ by providing high-quality sensing data, or $d$ by submitting low-quality ones.

In fact, the action of a player is driven by utility. We divide the whole sensing time into multiple intervals for the consideration of time-sensitive subtasks, and the action of each player is regarded unchanged during each interval. Then, the utility of the requestor at interval $t$ can be defined as follows:

$$
s_{r}^{t}=A^{r} \sum_{i=1}^{n_{a}} \sum_{j=1}^{b_{i}} x\left(w_{i j}^{t}\right)-B^{r} \sum_{i=1}^{n_{a}} \sum_{j=1}^{b_{i}} y\left(r_{i j}^{t}\right),
$$

where $n_{a}$ is the total number of target sensing regions, $b_{i}$ denotes the number of workers in sensing region $i \in$ $\left\{1, \cdots, n_{a}\right\}, w_{i j}^{t}$ refers to the action of worker $j$ in region $i$ at interval $t$, and $r_{i j}^{t}$ represents the action of the requestor against $w_{i j}^{t}$. In the above equation, the first term denotes the profit of the requestor obtained from the sensing data in all target regions ${ }^{2}$, while the second one represents her cost, i.e., the payment to the workers. Particularly, $A^{r}$ and $B^{r}>0$ are scale parameters, $x(\cdot)$ represents the profit of the requestor from each worker, and $y(\cdot)$ is the cost function related to the action of the requestor, i.e., the payment to each worker. Note that functions $x(\cdot)$ and $y(\cdot)$ here are in general forms and they will be assigned specific formulas in the experiment section. Also note that in the profit and cost

2. Here we consider that the requestor hires the exact number of workers to complete the MCS task without any redundancy, and thus her profit is proportional to the number of workers. In addition, even for the case of workers' submissions leading to the marginal utility diminishing problem for the requestor, the basic methodology to achieve the cost-efficient mobile crowdsensing remains unchanged. Thus we consider the summation form of the requestor's utility benefiting from all workers for the brevity of analysis in this paper. 
functions, the action $c$ results in a higher value while $d$ leads to a lower one.

Similarly, the utility of any worker $j$ in region $i$ at interval $t$ can be defined as

$$
s_{i j}^{t}=A^{i j} y\left(r_{i j}^{t}\right)-B^{i j} c_{i j}^{t}\left(w_{i j}^{t}\right),
$$

where $A^{i j}, B^{i j}>0$ are scale parameters, and $c_{i j}^{t}(\cdot)$ is the cost function of worker $j$ in region $i$ at interval $t$, which is higher with behavior $c$ and lower with $d$. The specific form of $c_{i j}^{t}(\cdot)$ will be exemplified in our experimental studies. Note that all $s_{r}^{t}$ and $s_{i j}^{t}\left(i \in\left\{1, \cdots, n_{a}\right\}, j \in\left\{1, \cdots, b_{i}\right\}\right)$ are non-negative, since otherwise no one would take part in the MCS.

In light of (1), the requestor should lay down a pricing strategy for every worker in each region at any interval. Let $n_{t}$ be the total number of sensing intervals. Then, the requestor needs to work out $n_{a} \times b_{i} \times n_{t}$ pricing strategies, which is a long haul for the requestor. Hence, there is a pressing need to study the pricing strategies for the requestor in a cost-efficient way. According to the utility functions shown in (1) and (2), we can derive the expected utilities of the requestor and any worker $j$ in region $i$ at interval $t$ as follows,

$$
\begin{gathered}
\Pi_{r}^{t}=A^{r} \sum_{i=1}^{n_{a}} \sum_{j=1}^{b_{i}} \tilde{x}\left(f_{i j}^{t}\right)-B^{r} \sum_{i=1}^{n_{a}} \sum_{j=1}^{b_{i}} \tilde{y}\left(h_{i j}^{t}\right), \\
\Pi_{i j}^{t}=A^{i j} \tilde{y}\left(h_{i j}^{t}\right)-B^{i j} \tilde{c}_{i j}^{t}\left(f_{i j}^{t}\right),
\end{gathered}
$$

where $f_{i j}^{t}$ and $h_{i j}^{t} \in[0,1]$ are respectively the probability for worker $j$ to choose $c$ in region $i$ at interval $t$ and that of the requestor when playing against this worker; $\tilde{x}(\cdot), \quad \tilde{y}(\cdot), \quad \tilde{c}_{i j}^{t}(\cdot)$ are monotonically increasing with the cooperation probability whose maximum and minimum are respectively equal to $x(c), y(c), c_{i j}^{t}(c)$ and $x(d), y(d), c_{i j}^{t}(d)$. Then we have $\frac{\partial \Pi_{r}^{t}}{\partial f_{i j}^{t}}=A^{r} \frac{\partial \tilde{x}}{\partial\left(f_{i j}^{t}\right)}>0$ and $\frac{\partial \Pi_{r}^{t}}{\partial h_{i j}^{t}}=-B^{r} \frac{\partial \tilde{y}}{\partial h_{i j}^{t}}<0$ since $A^{r}, B^{r}>0$ and $\tilde{x}(\cdot), \tilde{y}(\cdot)$ are both monotone increasing functions, which indicate that the expected utility of the requestor is increasing with the cooperation probability of each worker $f_{i j}^{t}$ and decreasing with her own cooperation probability $h_{i j}^{t}$. Through a similar analysis, we can find that the expected utility of worker $j$ in region $i$ at interval $t$ is in the opposite situation where a higher $h_{i j}^{t}$ and a lower $f_{i j}^{t}$ lead to a larger value.

Fig. 3 plots an example of the expected utility of the requestor brought by a worker and that of the worker, where $\tilde{x}\left(f_{i j}^{t}\right)=f_{i j}^{t}+2, \tilde{y}\left(h_{i j}^{t}\right)=h_{i j}^{t}+1, \tilde{c}_{i j}^{t}\left(f_{i j}^{t}\right)=0.5 f_{i j}^{t}+0.5$, and $A^{r}=6, B^{r}=2, A^{i j}=B^{i j}=1, n_{a}=4, b_{i}=1, j=1, i \in$ $\{1,2,3,4\}$. According to this figure, we can find that at the point $\left(f_{i j}^{t}, h_{i j}^{t}\right)=(1,0)$, the expected utility of the requestor is maximized while that of the worker is minimized.

Based on the above analysis, one can see that the requestor has to minimize the utility of the worker in order to maximize her own utility. However, it is not only time-consuming but also energy-consuming for the requestor to work out each pricing strategy to minimize every worker's expected utility in a one-by-one manner, which calls for cost-efficient operations. To that aim, we turn the idea of the worker-customized pricing strategy to that of
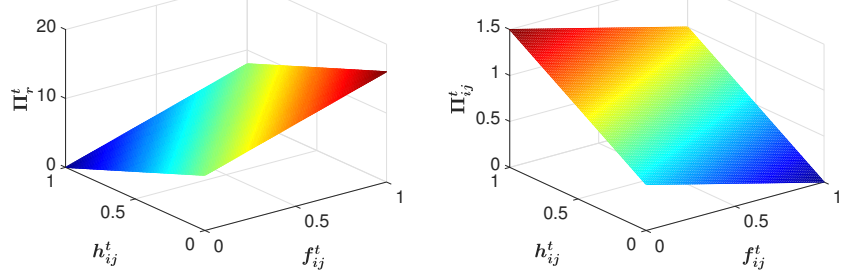

Fig. 3. Expected utilities of the requestor and the worker.

an interval-customized one. More specifically, the requestor can formulate a pricing strategy for each interval rather than each worker to minimize the expected utilities of the workers in all sensing regions. As a result, the number of pricing strategies the requestor should work out reduces from $n_{a} \times b_{i} \times n_{t}$ to $n_{t}$.

Let $r_{j}$ be the sum of the workers' expected utilities in all sensing regions at interval $j \in\left\{1,2, \ldots, n_{t}\right\}$. Then, the goal of our interval-customized pricing strategy is to minimize $\left\{r_{1}, r_{2}, \ldots, r_{n_{t}}\right\}$, where

$$
\left\{\begin{array}{c}
r_{1}=a_{11} \pi_{1}+a_{12} \pi_{2}+\cdots+a_{1 n_{a}} \pi_{n_{a}}, \\
r_{2}=a_{21} \pi_{1}+a_{22} \pi_{2}+\cdots+a_{2 n_{a}} \pi_{n_{a}}, \\
\vdots \\
r_{n_{t}}=a_{n_{t} 1} \pi_{1}+a_{n_{t} 2} \pi_{2}+\cdots+a_{n_{t} n_{a}} \pi_{n_{a}}
\end{array}\right.
$$

In the above equation, $a_{j i}, i \in\left\{1, \cdots, n_{a}\right\}, j \in\left\{1, \cdots, n_{t}\right\}$, is the number of workers in region $i$ at interval $j$, and $\pi_{i}$ is the expected utility of any worker in region $i$.

To realize a cost-efficient deal, an intuitive idea is to search for the equilibrium strategies for all players in their game, and then the optimal utility of the requestor is constrained by the actions of the workers. Differently, in this paper, we intend to realize a greedy goal that enables the requestor to minimize the utilities of the workers without any concern about the workers' actions. An intuitive idea to minimize any $r_{j}\left(j \in\left\{1,2, \ldots, n_{t}\right\}\right)$ is to enable the requestor to set its value to be the minimum. Such an idea is totally out of imagination since the expected utility of each player is not only decided by his/er own action but also dependent on those of the opponents in classic game theories. This drives us to resort to the ZD strategy [22], which was proposed by Press and Dyson and provided us with a revolutionary understanding of Markovian games. Taking advantage of ZD, a ZD player can unilaterally set the expected utility of its opponent or the ratio of their expected utilities, no matter what actions the opponent adopts. Hence, ZD strategies offer us the opportunities to achieve our goal.

However, it is costly to directly apply ZD strategies to our problem in that they are essentially worker-customized. To realize an interval-customized pricing strategy, the requestor needs to employ a homogeneous strategy to set the expected utilities of the workers throughout all sensing regions for a specific interval, so as to reduce the computational cost with the consideration of the spatiotemporal heterogeneity about the number of workers. This requires to extend ZD, which was originally designed 
for iterated two-player simultaneous games, so as to make it suitable for iterated multiple-player sequential games. More importantly, such an extension should empower the requestor to set a given value to the linear combination of the expected utilities of the workers in different regions. In other words, the requestor can make $r_{j}=\sum_{i=1}^{n_{a}} a_{j i} \pi_{i}$ $\left(j \in\left\{1,2, \ldots, n_{t}\right\}\right)$ hold no matter how the workers act. Due to its function, we call this extended ZD strategy the combinatorial pinning ZD strategy. In next section, we shall detail the method on how to realize this ZD strategy, which can not only be utilized to solve our problem, but also enrich the theoretical hierarchy of ZD strategies, broadening their application scope.

\section{Combinatorial Pinning ZD Strategy}

The proposed combinatorial pinning ZD strategy is applicable to an iterated multiple-player sequential game, which contains two types of players, namely first-move players (leaders) and second-move players (followers). Here we focus on a scenario with one leader, denoted as $n_{0}$, and $N$ followers, indicated by $\left\{n_{1}, n_{2}, \cdots, n_{N}\right\}$. Each player has two possible actions, i.e., cooperation $(c)$ and defection (d). Thus, there could be in total $\eta=2^{(N+1)}$ game states, denoted by

$$
e_{0} e_{1} \cdots e_{N}=\{\underbrace{c \overbrace{c c \cdots c}^{N}}_{g_{1}}, \underbrace{c \overbrace{c c \cdots d}^{N-1}}_{g_{2}}, \cdots, \underbrace{d \overbrace{d d \cdots d}^{N}}_{g_{\eta}}\},
$$

where $e_{0}$ and $e_{i}$ respectively represent the actions of leader $n_{0}$ and follower $n_{i}$. For brevity, we denote the set of game states as $G=\left\{g_{1}, g_{2}, \cdots, g_{\eta}\right\}$.

It is reasonable for the leader to choose its action according to the game result of the previous round. This assumption is consistent with the conclusion in [22], which states that a short-memory player has no disadvantage compared to a long-memory one. Thus, following the order of game states listed in (4), we define the strategy of the leader as $\mathbf{p}=\left(p_{1}, p_{2}, \cdots, p_{\eta}\right)$, where each element $p_{i}$ is the conditional probability of choosing action $c$ when the game state of the previous round is $g_{i}$; accordingly, $1-p_{i}$ is the conditional probability of choosing $d$ given the previous game state of $g_{i}$.

For any follower $n_{i}, i \in\{1,2, \cdots, N\}$, since it moves after $n_{0}$, it is rational to react based on the action of the leader in the same round. As the action $e_{0}$ of the leader could be either $c$ or $d$, we define the strategy of follower $n_{i}$ as $\mathbf{q}^{i}=\left(q_{1}^{i}, q_{2}^{i}\right)$, where $q_{1}^{i}\left(q_{2}^{i}\right)$ refers to the conditional probability of choosing $c$ when the leader performs $c(d)$ in the current round. Thus, $1-q_{1}^{i}$ and $1-q_{2}^{i}$ are respectively the defection probabilities when the leader cooperates or defects in the current round.

Given the above definitions of $\mathbf{p}$ and $\mathbf{q}^{i}, i \in\{1, \cdots, N\}$, one can formulate the state transition matrix of the game as

$$
\mathbf{M}=\left[M_{u v}\right]_{\eta \times \eta},
$$

where each element $M_{u v}$ denotes the transition probability from the last game state $g_{u}$ to the current state $g_{v}$. It can be calculated as follows,

$$
M_{u v}=P \prod_{i=1}^{N} Q_{i}
$$

with $P$ and $Q_{i}$ being the action probabilities of leader $n_{0}$ and follower $n_{i}$ at state $g_{v}$. In particular, we denote the action of $n_{0}$ in state $g_{v}$ as $z_{0}$, which equals 1 when $e_{0}=c$ and 0 when $e_{0}=d$. Similarly, the action of $n_{i}$ at state $g_{v}$ is denoted as $z_{i}$, with value 1 when $e_{i}=c$ and 0 otherwise. Thus, we can calculate $P$ and $Q_{i}$ as follows:

$$
\begin{gathered}
P=\left(p_{u}\right)^{z_{0}}\left(1-p_{u}\right)^{1-z_{0}}, \\
Q_{i}=\left\{\left(q_{1}^{i}\right)^{z_{0}}\left(q_{2}^{i}\right)^{1-z_{0}}\right\}^{z_{i}}\left\{1-\left(q_{1}^{i}\right)^{z_{0}}\left(q_{2}^{i}\right)^{1-z_{0}}\right\}^{1-z_{i}} .
\end{gathered}
$$

Note that the calculation of $P$ in (6) depends on $p_{u}$, i.e., the cooperation probability of $n_{0}$ when the last game state is $g_{u}$, which is resulted from the assumption that the leader makes decisions based on the game state of the previous round. Clearly, when the current game state $g_{v}$ indicates that the action of $n_{0}$ is $c, P=p_{u}$; otherwise, $P=1-p_{u}$. While the computation of $Q_{i}$ in (7) seems to be more complicated, which is resulted from the follower's property of relying on the action of the leader in the current round. When the current action of the leader is $c, q_{1}^{i}$ functions actively, which can be combined with the action requirement of the current state $g_{v}$ to finally determine the value of $Q_{i}$, i.e., $q_{1}^{i}$ or $1-q_{1}^{i}$; otherwise, it is $q_{2}^{i}$ that functions.

Next, we define a matrix $\mathbf{M}^{\prime}=\mathbf{M}-\mathbf{I}$ with $\mathbf{I}$ denoting the identity matrix with the same size as $\mathbf{M}$, i.e., $\eta \times \eta$. If the stable vector of the state transition matrix $\mathbf{M}$ is denoted as $\mathbf{v}$, we have $\mathbf{v}^{\top} \mathbf{M}=\mathbf{v}^{\top}$, which is also equivalent to $\mathbf{v}^{\top} \mathbf{M}^{\prime}=0$. When we apply the Cramer's rule to matrix $\mathbf{M}^{\prime}$, we have $\operatorname{Adj}\left(\mathbf{M}^{\prime}\right) \mathbf{M}^{\prime}=\operatorname{det}\left(\mathbf{M}^{\prime}\right) \mathbf{I}=0$ with $\operatorname{Adj}\left(\mathbf{M}^{\prime}\right)$ denoting the adjugate matrix. Combining the above two equations, we can conclude that $\mathbf{v}$ is proportional to each row of $\operatorname{Adj}\left(\mathbf{M}^{\prime}\right)$. Let $\mathbf{M}^{\prime}=\left[\mathbf{M}_{1}, \mathbf{M}_{2}, \cdots, \mathbf{M}_{\eta}\right]$, where $\mathbf{M}_{i}=\left[M_{1 i}, M_{2 i}, \cdots, M_{\eta i}\right]^{\top}$ is a vector consisting of the $i$ th column of $\mathbf{M}^{\prime}$. Thus, for any vector $\mathbf{f}=\left[f_{1}, f_{2}, \cdots, f_{\eta}\right]^{\top}$, we can compute its dot product with the stable vector $\mathbf{v}$ as

$$
\mathbf{v} \cdot \mathbf{f}=\operatorname{det}\left[\mathbf{M}_{1}, \mathbf{M}_{2}, \cdots, \mathbf{M}_{\eta-1}, \mathbf{f}\right] .
$$

As we all know, elementary transformations of a matrix cannot change its determinant value. Thus, we can first find a column in $\mathbf{M}^{\prime}$ corresponding to a particular current game state which is consisted of the leader's cooperation and all followers' defection, i.e., $e_{0} e_{1} \cdots e_{N}=c d \cdots d$. It is easy to see that this column is the $\frac{\eta}{2}$ th element of $\mathbf{M}^{\prime}$, following the order in the game state set $G$, which can be denoted as $\mathbf{M}_{\frac{\eta}{2}}=\left[p_{1} \prod_{i=1}^{N}\left(1-q_{1}^{i}\right), p_{2} \prod_{i=1}^{N}\left(1-q_{1}^{i}\right), \cdots, p_{\eta} \prod_{i=1}^{N}(1-\right.$ $\left.\left.q_{1}^{i}\right)\right]^{\top}$. Next, we conduct an elementary column operation on matrix $\mathbf{M}^{\prime}$ by adding all columns before the $\frac{\eta}{2}$ one, i.e., from the first column to the $\left(\frac{\eta}{2}-1\right)$ th one, to the $\frac{\eta}{2}$ th column. By doing this, one can easily calculate the updated value of the $\frac{\eta}{2}$ th column as follows, denoted by $\hat{\mathbf{M}}_{\frac{\eta}{2}}$,

$$
\hat{\mathbf{M}}_{\frac{\eta}{2}}=\left[p_{1}-1, p_{2}-1, \cdots, p_{\frac{\eta}{2}}-1, p_{\frac{\eta}{2}+1}, \cdots, p_{\eta}\right]^{\top} .
$$

Therefore, the dot product between $\mathbf{v}$ and $\mathbf{f}$ turns to be

$$
\begin{aligned}
\mathbf{v} \cdot \mathbf{f} & =\operatorname{det}\left[\mathbf{M}_{1}, \cdots, \hat{\mathbf{M}} \frac{\eta}{2}, \cdots, \mathbf{M}_{\eta-1}, \mathbf{f}\right] \\
& =\operatorname{det}\left[\begin{array}{ccccc}
p_{1} \prod_{i=1}^{N} q_{1}^{i}-1 & \cdots & p_{1}-1 & \cdots & f_{1} \\
\vdots & \ddots & \vdots & \ddots & \vdots \\
p_{\frac{\eta}{2}} \prod_{i=1}^{N} q_{1}^{i} & \cdots & p_{\frac{\eta}{2}}-1 & \cdots & f_{\frac{\eta}{2}} \\
\vdots & \ddots & \vdots & \ddots & \vdots \\
p_{\eta} \prod_{i=1}^{N} q_{1}^{i} & \cdots & p_{\eta} & \cdots & f_{\eta}
\end{array}\right] .
\end{aligned}
$$


Clearly, the $\frac{\eta}{2}$ th column of the matrix, i.e., $\hat{\mathbf{M}}_{\frac{\eta}{2}}$, is fully decided by the strategy of the leader. In this case, for any constant parameter $\phi \neq 0$, when the leader sets its strategy p to make $\hat{\mathbf{M}}_{\frac{\eta}{2}}=\phi \mathbf{f}$, the $\frac{\eta}{2}$ th column is proportional to the last column, leading to the zero determinant value in (10), i.e., $\mathbf{v} \cdot \mathbf{f}=0$.

Leveraging on the stable vector of the state transition matrix in the Markov process, we can calculate the expected payoff of each player $n_{i}(i \in\{0,1,2, \cdots, N\})$, denoted by $E_{i}$, as follows,

$$
E_{i}=\frac{\mathbf{v} \cdot \mathbf{S}_{i}}{\mathbf{v} \cdot \mathbf{1}}=\frac{\operatorname{det}\left[\mathbf{M}_{1}, \cdots, \mathbf{M}_{\eta-1}, \mathbf{S}_{i}\right]}{\operatorname{det}\left[\mathbf{M}_{1}, \cdots, \mathbf{M}_{\eta-1}, \mathbf{1}\right]},
$$

where $\mathbf{S}_{i}=\left\{S_{i}^{1}, S_{i}^{2}, \cdots, S_{i}^{\eta}\right\}$ is the payoff vector of player $n_{i}$ with each element $S_{i}^{j}$ corresponding to the payoff of game state $g_{j}, j \in\{1,2, \cdots, \eta\}$, and 1 refers to a vector consisting of $\eta$ components of 1 . In this situation, when we calculate a linear combination of their expected payoffs, it turns to be

$$
\begin{array}{r}
\alpha_{0} E_{0}+\sum_{i=1}^{N} \alpha_{i} E_{i}-\gamma=\frac{\mathbf{v} \cdot\left(\alpha_{0} \mathbf{S}_{0}+\sum_{i=1}^{N} \alpha_{i} \mathbf{S}_{i}-\gamma \mathbf{1}\right)}{\mathbf{v} \cdot \mathbf{1}} \\
=\frac{\operatorname{det}\left[\mathbf{M}_{1}, \cdots, \mathbf{M}_{\eta-1}, \alpha_{0} \mathbf{S}_{0}+\sum_{i=1}^{N} \alpha_{i} \mathbf{S}_{i}-\gamma \mathbf{1}\right]}{\operatorname{det}\left[\mathbf{M}_{1}, \cdots, \mathbf{M}_{\eta-1}, \mathbf{1}\right]},
\end{array}
$$

where $\alpha_{i}(i \in\{0,1,2, \cdots, N\})$ is a constant. According to the dot product computation in (10), we can find that when $\hat{\mathbf{M}}_{\frac{\eta}{2}}=\phi\left(\alpha_{0} \mathbf{S}_{0}+\sum_{i=1}^{N} \alpha_{i} \mathbf{S}_{i}-\gamma \mathbf{1}\right)(\phi \neq 0)$, the numerator in the above equation becomes zero. Thus we have

$$
\alpha_{0} E_{0}+\sum_{i=1}^{N} \alpha_{i} E_{i}-\gamma=0
$$

Therefore, with an appropriate setting of its strategy, the leader can control the linear relationship between its expected payoff and those of the followers. Particularly, when $\alpha_{0}=0$, the above equation becomes

$$
\sum_{i=1}^{N} \alpha_{i} E_{i}=\gamma
$$

and in this case, the corresponding strategy $\mathbf{p}$ of the leader should meet the condition

$$
\hat{\mathbf{M}}_{\frac{\eta}{2}}=\phi\left(\sum_{i=1}^{N} \alpha_{i} \mathbf{S}_{i}-\gamma \mathbf{1}\right)(\phi \neq 0) .
$$

By adopting the strategy satisfying condition (13), the leader can unilaterally set the weighted sum of the expected payoffs of all followers to be a fixed value, no matter what actions these followers adopt. Hence, we call this strategy a combinatorial pinning $Z D$ strategy, which is applicable to iterated multiple-player sequential games. Compared with the original ZD strategies derived for iterated twoplayer simultaneous games, the combinatorial pinning ZD strategy has its characteristic of only allowing the leader to unilaterally set the expected payoffs of others (followers). In other words, the players are equal in the original ZD strategies while the leader dominates in the combinatorial pinning ZD strategy. One can see that the combinatorial pinning ZD strategy can not only enrich the theoretical hierarchy of ZD strategies but also be used to solve the problem we formulate in Section 3, which will be detailed in the next section.

\section{Cost-Efficient MCS}

In this section, we employ the combinatorial pinning ZD strategy to realize a cost-efficient MCS with spatial-temporal awareness. Our main idea is to design a set of intervalcustomized pricing strategies for the requestor, namely $\rho=\left\{\tilde{\mathbf{p}}^{1}, \tilde{\mathbf{p}}^{2}, \cdots, \tilde{\mathbf{p}}^{n_{t}}\right\}$, with each element $\tilde{\mathbf{p}}^{j}$ being able to minimize the total expected utilities of all workers at a certain interval $j\left(j \in\left\{1,2, \ldots, n_{t}\right\}\right)$. Next, we explain how to obtain each $\tilde{\mathbf{p}}^{j}$. Let the strategy of the worker in region $i$ at interval $j$ be $\tilde{\mathbf{q}}^{i j}=\left(\tilde{q}_{1}^{i j}, \tilde{q}_{2}^{i j}\right)$, where $\tilde{q}_{1}^{i j}\left(\tilde{q}_{2}^{i j}\right)$ is the worker's conditional probability of performing $c$ given the requestor's action $c(d)$ in the current round. Thus, we can construct the following state transition matrix similar to (5):

$$
\mathbf{H}=\left[\begin{array}{ccc}
\tilde{p}_{1}^{j} \prod_{i=1}^{n_{a}} \tilde{q}_{1}^{i j} & \ldots & \left(1-\tilde{p}_{1}^{j}\right) \prod_{i=1}^{n_{a}}\left(1-\tilde{q}_{2}^{i j}\right) \\
\tilde{p}_{2}^{j} \prod_{i=1}^{n_{a}} \tilde{q}_{1}^{i j} & \cdots & \left(1-\tilde{p}_{2}^{j}\right) \prod_{i=1}^{n_{a}}\left(1-\tilde{q}_{2}^{i j}\right) \\
\vdots & \ddots & \vdots \\
\tilde{p}_{\zeta}^{j} \prod_{i=1}^{n_{a}} \tilde{q}_{1}^{i j} & \cdots & \left(1-\tilde{p}_{\zeta}^{j}\right) \prod_{i=1}^{n_{a}}\left(1-\tilde{q}_{2}^{i j}\right)
\end{array}\right]
$$

where $\zeta$ is the number of game states between the requestor and the workers. Next, with similar calculations presented in the previous section, we can find that the $\frac{\zeta}{2}$ th column in the adjugate matrix of $\mathbf{H}$, denoted as $\hat{\mathbf{H}}_{\frac{\zeta}{2}}=\left[\tilde{p}_{1}^{j}-\right.$ $\left.1, \tilde{p}_{2}^{j}-1, \cdots, \tilde{p}_{\frac{\zeta}{2}}^{j}-1, \tilde{p}_{\frac{\zeta}{2}+1}^{j}, \cdots, \tilde{p}_{\zeta}^{j}\right]^{\top}$, can be unilaterally determined by the requestor's strategy. Based on this, the requestor can set $r_{j}=\sum_{i=1}^{n_{a}} a_{j i} \pi_{i}$ to any value as long as her strategy is meaningful, i.e., $\tilde{p}_{k}^{j} \in[0,1]$, where $k \in\{1,2, \cdots, \zeta\}$.

Hence, the problem of employing the combinatorial pinning ZD strategy to realize a cost-efficient deal in MCS with cost-efficient strategies can be addressed by solving the following constrained optimization problem for each $j \in\left\{1,2, \ldots, n_{t}\right\}$,

$$
\begin{array}{ll}
\min & r_{j}=\sum_{i=1}^{n_{a}} a_{j i} \pi_{i} \\
\text { s.t. } & \left\{\begin{array}{l}
\mathbf{0} \leq \tilde{\mathbf{p}}^{j} \leq \mathbf{1}, \\
\hat{\mathbf{H}}_{\frac{\zeta}{2}}=\phi\left(\sum_{i=1}^{n_{a}} a_{j i} \mathbf{F}_{j i}-r_{j} \mathbf{1}\right), \\
\phi \neq 0
\end{array}\right.
\end{array}
$$

where $\mathbf{F}_{j i}=\left[F_{j i}^{1}, F_{j i}^{2}, \cdots, F_{j i}^{\zeta}\right]$ is the utility vector of any worker in region $i$ at interval $j$ with each $F_{j i}^{k}(k \in$ $\{1,2, \cdots, \zeta\})$ being calculated in light of $(2)^{3}$. According to the last constraint, we can solve the above optimization problem by considering the following two cases.

3. We don't consider the index of the workers in the same region when computing the expected utility of any worker. 
Case 1: $\phi>0$. To meet the constraint $\tilde{\mathbf{p}}^{j} \geq \mathbf{0}$, we can get an upper bound of $r_{j}$ as follows:

$$
\begin{aligned}
& r_{j}^{\max }= \min \left(\beta_{k}\right), \forall k \in\{1,2, \cdots, \zeta\}, \\
& \beta_{k}=\left\{\begin{array}{l}
\frac{1}{\phi}+\sum_{i=1}^{n_{a}} a_{j i} F_{j i}^{k}, k=1,2, \cdots, \frac{\zeta}{2}, \\
\sum_{i=1}^{n_{a}} a_{j i} F_{j i}^{k}, k=\frac{\zeta}{2}+1, \cdots, \zeta .
\end{array}\right.
\end{aligned}
$$

While to satisfy $\tilde{\mathbf{p}}^{j} \leq \mathbf{1}$, we can obtain a lower bound of $r_{j}$,

$$
\begin{aligned}
& r_{j}^{\text {min }}=\max \left(\beta_{l}\right), \forall l \in\{\zeta+1, \zeta+2, \cdots, 2 \zeta\}, \\
& \beta_{l}=\beta_{k+\zeta}=\left\{\begin{array}{l}
\sum_{i=1}^{n_{a}} a_{j i} F_{j i}^{k}, k=1,2, \cdots, \frac{\zeta}{2}, \\
-\frac{1}{\phi}+\sum_{i=1}^{n_{a}} a_{j i} F_{j i}^{k}, k=\frac{\zeta}{2}+1, \cdots, \zeta .
\end{array}\right.
\end{aligned}
$$

Since only if $r_{j}^{\min } \leq r_{j}^{\max }$ can $r_{j}$ have a feasible solution, which is equal to $\max \left(\beta_{l}\right) \leq \min \left(\beta_{k}\right), \forall k \in$ $\{1,2, \cdots, \zeta\}, \forall l \in\{\zeta+1, \zeta+2, \cdots, 2 \zeta\}$. If there exists $\phi>0$ satisfying the above constraint, the minimum value of $r_{j}$ is

$$
\begin{aligned}
r_{j}^{\min }=\max \{ & \sum_{i=1}^{n_{a}} a_{j i} F_{j i}^{1}, \cdots, \sum_{i=1}^{n_{a}} a_{j i} F_{j i}^{\frac{\zeta}{2}}, \\
& \left.-\frac{1}{\phi}+\sum_{i=1}^{n_{a}} a_{j i} F_{j i}^{\frac{\zeta}{2}+1}, \cdots,-\frac{1}{\phi}+\sum_{i=1}^{n_{a}} a_{j i} F_{j i}^{\zeta}\right\} .
\end{aligned}
$$
have

Case 2: $\phi<0$. Considering the constraint $\tilde{\mathbf{p}}^{j} \geq \mathbf{0}$, we

$$
r_{j}^{\min }=\max \left(\beta_{k}\right), \forall k \in\{1,2, \cdots, \zeta\} .
$$

And according to $\tilde{\mathbf{p}}^{j} \leq \mathbf{1}$, we have

$$
r_{j}^{\max }=\min \left(\beta_{l}\right), \forall l \in\{\zeta+1, \zeta+2, \cdots, 2 \zeta\} .
$$

Similarly, $r_{j}$ has a feasible solution only when $r_{j}^{\text {min }} \leq r_{j}^{\text {max }}$ holds, i.e., $\max \left(\beta_{k}\right) \leq \min \left(\beta_{l}\right), \forall k \in\{1,2, \cdots, \zeta\}, \forall l \in$ $\{\zeta+1, \zeta+2, \cdots, 2 \zeta\}$. If we can find $\phi<0$ that makes this constraint always hold, we can get the minimum of $r_{j}$ as,

$$
\begin{aligned}
r_{j}^{\min }=\max \left\{\frac{1}{\phi}+\sum_{i=1}^{n_{a}} a_{j i} F_{j i}^{1}, \cdots, \frac{1}{\phi}+\sum_{i=1}^{n_{a}} a_{j i} F_{j i}^{\frac{\zeta}{2}},\right. \\
\left.\sum_{i=1}^{n_{a}} a_{j i} F_{j i}^{\frac{\zeta}{2}+1}, \cdots, \sum_{i=1}^{n_{a}} a_{j i} F_{j i}^{\zeta}\right\} .
\end{aligned}
$$

In summary, the requestor can unilaterally set the total expected utilities of the workers in all regions at any interval $j$ to be the lowest one, i.e., $r_{j}^{\min }$, when she adopts the combinatorial pinning ZD strategy $\tilde{\mathbf{p}}^{j}=\left(\tilde{p}_{1}^{j}, \tilde{p}_{2}^{j}, \cdots, \tilde{p}_{\zeta}^{j}\right)$ satisfying $\hat{\mathbf{H}}_{\frac{\zeta}{2}}=\phi\left(\sum_{i=1}^{n_{a}} \alpha_{i} \mathbf{F}_{j i}-r_{j}^{\text {min }} \mathbf{1}\right)$, i.e.,

$$
\tilde{p}_{k}^{j}=\left\{\begin{array}{l}
\phi\left(\sum_{i=1}^{n_{a}} a_{j i} F_{j i}^{k}-r_{j}^{m i n}\right)+1, k=1,2, \cdots, \frac{\zeta}{2}, \\
\phi\left(\sum_{i=1}^{n_{a}} a_{j i} F_{j i}^{k}-r_{j}^{m i n}\right), k=\frac{\zeta}{2}+1, \cdots, \zeta .
\end{array}\right.
$$

By this means, the requestor can derive the set of interval-customized pricing strategies, namely $\rho=$ $\left\{\tilde{\mathbf{p}}^{1}, \tilde{\mathbf{p}}^{2}, \cdots, \tilde{\mathbf{p}}^{n_{t}}\right\}$, to minimize the total expected utilities $\left\{r_{1}, r_{2}, \cdots, r_{n_{t}}\right\}$. Hence, the corresponding expected utility of any worker in each region at every interval can be calculated by solving (3), which indicates the potential of our proposed combinatorial pinning ZD strategy to transfer the setting of individual-customized expected utility to a category-customized one. Let $\mathbf{A}=\left[a_{j i}\right]_{n_{t} \times n_{a}}, \boldsymbol{\Pi}=$ $\left[\pi_{1}, \pi_{2}, \cdots, \pi_{n_{a}}\right]^{\top}$ and $\mathbf{r}=\left[r_{1}, r_{2}, \cdots, r_{n_{t}}\right]^{\top}$; thus the equation group in (3) can be denoted as $\mathbf{A} \cdot \boldsymbol{\Pi}=\mathbf{r}$. As the number of workers in each sensing region and time interval varies, we consider the following three cases.

Case 1: If $n_{t}=n_{a}$, the coefficient matrix $\mathbf{A}$ is a square. We have the following subcases with respect to different determinant values of $\mathbf{A}$ :

- When $\operatorname{det}(\mathbf{A}) \neq 0$, according to the Cramer's rule, we can get the result of $\boldsymbol{\Pi}$ as $\pi_{i}=\frac{\operatorname{det}\left(\mathbf{A}_{i}\right)}{\operatorname{det}(\mathbf{A})}, i=$ $1, \cdots, n_{a}$, where $\mathbf{A}_{i}$ is the matrix formed by substituting the $i$ th column of $\mathbf{A}$ with the vector $\mathbf{r}$.

- When $\operatorname{det}(\mathbf{A})=0$ and $\operatorname{rank}(\mathbf{A})=\operatorname{rank}(\mathbf{A}, \mathbf{r})$, there exist multiple solutions for $\boldsymbol{\Pi}$, from which the requestor can select one.

- When $\operatorname{det}(\mathbf{A})=0$ and $\operatorname{rank}(\mathbf{A})<\operatorname{rank}(\mathbf{A}, \mathbf{r})$, there exists no solution for $\boldsymbol{\Pi}$, which means that the requestor cannot efficiently set the expected utilities of the workers. In this case, the requestor can redivide the sensing regions and intervals to find suitable $n_{t}$ and $n_{a}$ such that the optimal strategies of the requestor and the expected utility of any worker can be derived.

Case 2: If $n_{a}>n_{t}$, there exist multiple solutions for (3); and the requestor can select any one to reach her goal.

Case 3: If $n_{a}<n_{t}$, no solution exists unless $\operatorname{rank}(\mathbf{A}, \mathbf{r})=n_{a}$, which can be addressed with the discussions in Case 1.

\section{EXPERIMENTAL EVALUATION}

In this section, we evaluate the performance of the proposed cost-efficient MCS based on a real-world dataset. The dataset contains the long-term check-in data in the New York City collected by Foursquare from Apr. 3, 2012 to Feb. 16, 2013 [12], which includes 227,428 records generated from 1,083 users at more than 40,000 locations. In our experiment, we utilize this check-in dataset to simulate a mobile crowdsensing scenario where the visitors are workers to undertake a large-scale sensing task assigned by the requestor, and implement it using Matlab R2018a on a machine with $2.3 \mathrm{GHz}$ Intel Core i5 processor. To that aim, we divide the New York City into four target sensing regions, i.e., $n_{a}=4$, and $n_{t}$ is also set to be 4 . Besides, for the utility function of the requestor, we set $A^{r}=0.5$ and $B^{r}=0.1$, the profit function $x(c)=1$ and $x(d)=0.5$, and the cost function $y(c)=2$ and $y(d)=1$; while for those of the worker $j$ in region $i(i, j \in\{1,2,3,4\})$, we set $A^{i j}=B^{i j}=1$, and the cost function $c_{i j}(c)=1$ and $c_{i j}(d)=0.5$. Each experiment is repeated 30 times to get the average result for statistical confidence. It is worth noting that we also test other parameter settings, which present very similar results; thus we omit them to avoid redundancy. 
In order to verify the effectiveness of our proposed combinatorial pinning ZD (CPZD) strategy based scheme, we compare it with three other classical strategies. To be specific, we simulate the game process between the requestor and the workers in 100 rounds, where the requestor adopts CPZD, all-cooperation (ALLC), alldefection (ALLD), and random (Random) strategies, while the workers take ALLC, ALLD, Random, tit-for-tat (TFT), and mixed (Mixed) strategies. A player adopting ALLC strategy always cooperates no matter what the opponent behaves; on the contrary, an ALLD player always defects. With Random strategy, a player cooperates with the probability of 0.5 ; a TFT player follows the choice of the opponent in the previous round. We also consider the situation of Mixed strategy where the workers in each region adopt a specific strategy chosen from ALLC, ALLD, Random, and TFT.

We first evaluate our proposed strategy for the total expected utility optimization at a specific interval. To that aim, we utilize the check-in data during 18:00 to 19:00 on Apr. 3, 2012 where the distribution of the visitors (workers) in different regions is $\left[a_{11}, a_{12}, a_{13}, a_{14}\right]=[11,43,13,18]$. And we set $\phi=-0.001$ to meet the requirement of $r_{1}^{\min } \leq r_{1}^{\max }$ as mentioned in Section 5. Fig. 4 plots the total expected utilities of the workers in each round when the requestor adopts the CPZD strategy and the workers employ different strategies. To clearly display the evolution process, we only present the results in the first 20 rounds. It is clear that no matter what kind of strategy the workers adopt, their total expected utilities finally converge to a fixed value, verifying the power of the proposed combinatorial pinning ZD strategy. Figs. 5 (a)(b)(c)(d) present the final total expected utilities of the workers when the requestor takes CPZD, ALLC, ALLD, and Random strategies, respectively. By comparing Fig. 5(a) with the other three figures, one can easily find that the total expected utilities of the workers change with the worker's own strategy in the cases of the requestor adopting ALLC, ALLD and Random strategies, and that they are mostly larger than the corresponding values when the requestor adopts the CPZD strategy except for the ALLD case. However, the requestor is unwise to adopt an ALLD strategy in real life since she would suffer from a bad reputation and is hard to recruit workers in the future. Besides, on average, the running time of our proposed CPZD strategy playing against ALLC, ALLD, Random, TFT and Mixed strategies in a specific interval are respectively $1.8 \mathrm{~ms}, 1.0 \mathrm{~ms}, 2.9 \mathrm{~ms}, 2.4 \mathrm{~ms}$ and $4.0 \mathrm{~ms}$.

Next, we investigate the effectiveness of our proposed strategy for multi-interval optimization. Similarly, we make use of the check-in data from 18:00 to 22:00 in Apr. 3, 2012, where the time interval is set to be one hour, and the distributions of the workers in the four regions are given by $\left[a_{j i}\right]_{4 \times 4}$. To be specific, $\left[a_{11}, a_{12}, a_{13}, a_{14}\right]=[11,43,13,18]$, $\left[a_{21}, a_{22}, a_{23}, a_{24}\right]=[26,35,12,14],\left[a_{31}, a_{32}, a_{33}, a_{34}\right]=$ $[20,47,16,16]$, and $\left[a_{41}, a_{42}, a_{43}, a_{44}\right]=[42,60,18,17]$. With the same parameter and strategy settings mentioned above, we plot the simulation results in Figs. 6 and 7. Fig. 6 presents the time-varying total expected utilities of the workers with ALLC, ALLD, Random, TFT, and Mixed strategies, against the requestor adopting the proposed CPZD strategy. For clarity, we also focus on the first 20 rounds of the game. It is

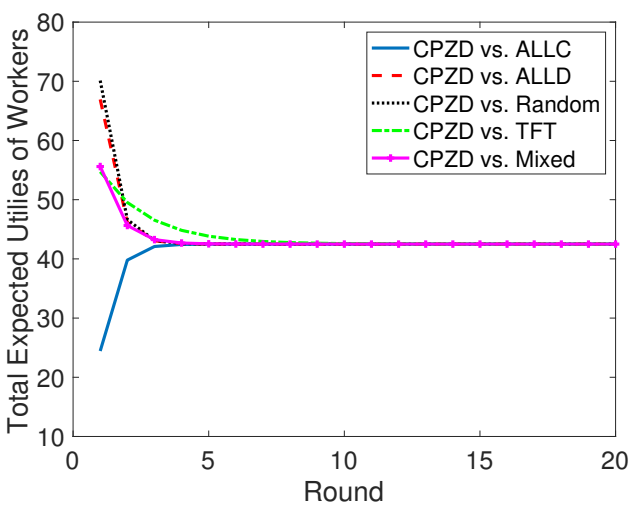

Fig. 4. Evolution of the total expected utilities of the workers in one interval.

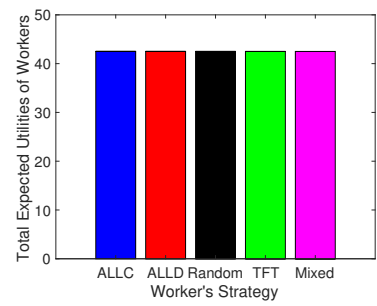

(a) CPZD

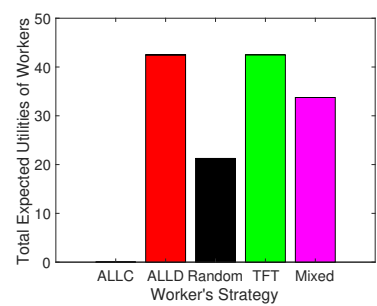

(c) ALLD

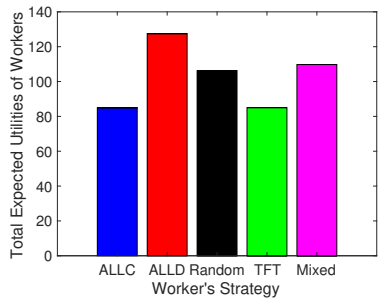

(b) ALLC

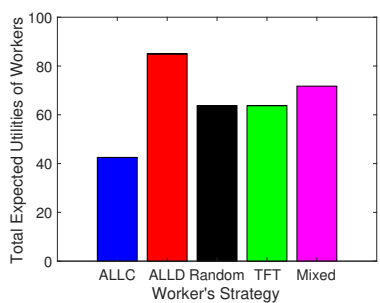

(d) Random
Fig. 5. Total expected utilities of the workers in a specific interval.

obvious the total utilities of the workers always converge to certain values whatever strategy the workers adopt. And Fig. 7 displays the total utilities of the workers in each interval. By comparing the results in Fig. 7(a) and those of the other three figures, we can conclude that our proposed CPZD strategy based method can help the requestor to always set the total expected utilities of the workers to be a stably minimum level except for the situation with an irrational requestor adopting the ALLD strategy. And the average running time of CPZD strategy of the requestor playing against ALLC, ALLD, Random, TFT, and Mixed strategies of the workers in four intervals are $5.7 \mathrm{~ms}, 4.3$ $\mathrm{ms}, 10.0 \mathrm{~ms}, 8.7 \mathrm{~ms}$, and $14.5 \mathrm{~ms}$, respectively.

It is worth noting that the above performance comparisons involve no incentive-based schemes because it is inappropriate to do that for the following reasons. First, the main goal of incentive mechanisms is different from ours, where the incentive-based ones mainly aim at incentivizing wide participation of workers or eliciting highquality submissions from workers, while we intend to figure out how to achieve a cost-efficient deal with a cost-efficient strategy for the requestor. Second, the modeling system of 


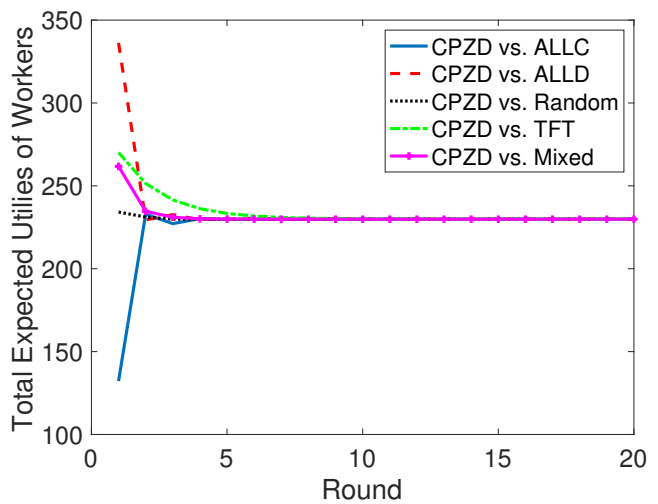

Fig. 6. Evolution of the total expected utilities of the workers in all intervals.

the incentive-based mechanisms is distinct from our model. To be specific, several auction-based incentive mechanisms define the actions of workers as the bidding price for completing the sensing task, and others regard the effort levels of the workers as their individual actions in a discrete manner; while in our model, we consider any worker may exert cooperation action by providing high-quality sensing result with a continuous probability, which is more general.

Finally, we study how the number of workers affects the minimum expected utilities of all workers in each interval and the strategy of the requestor according to our proposed scheme. The same check-in data and parameter settings mentioned above are adopted, and hence $\operatorname{det}(\mathbf{A}) \neq 0$, which means that we can get a unique solution of $\boldsymbol{\Pi}$. In Figs. 8 and 9 , we respectively adjust the values of each row and column of $\mathbf{A}$, making them twice, three times, and four times of the original ones. As shown in Fig. 8, the minimum expected utilities of all workers in different regions at interval $j$, i.e., $r_{j}$, increase with the values of the $j$ th row in the coefficient matrix $\mathbf{A}$, i.e., the number of workers in different regions at interval $j$, while other $r_{i}(i \neq j)$ has no change. It is consistent with the fact that more workers in an interval make the requestor set higher expected utilities in total. While in Fig. 9, it is easy to find that the values of all $r_{j}$ increase with the value of any column, i.e., the number of workers in a specific region at different intervals, which is also reasonable since more workers can bring more expected utilities.

Similarly, we plot the strategy of the requestor in each interval changing with the values of each row and column of $\mathbf{A}$ in Figs. 10 and 11, respectively. It is worth mentioning that the requestor's strategy in interval $j$, i.e., $\tilde{\mathbf{p}}^{j}$, has 32 components because there are one leader (the requestor) and four followers (workers in four different sensing regions), resulting in $\zeta=2^{5}=32$. In general, the requestor tends to be more cooperative when she cooperates in the last round but less cooperative when she defects in the previous game state. And in Fig. 10, it is easy to find that $\tilde{\mathbf{p}}^{j}$ decreases as the values of the $j$ th row in $\mathbf{A}$ increase, which is because the increasing number of workers in a specific interval reduces the requestor's demand of highquality sensing from one individual worker, resulting in her provision of less payment. With the same underlying reason, the cooperation probability of the requestor also slightly decreases with the increase of the column values as shown in Fig. 11.

\section{Conclusion and Future Research}

As a practice of sharing economy, MCS can exploit the off-the-shelf sensing resources economically and efficiently. The state-of-the-art studies ignore the dual cost-efficient demands, i.e., realizing a cost-efficient deal with a costefficient strategy, in large-scale sensing tasks. Hence, we propose a combinatorial pinning ZD strategy to empower the requestor to employ a single strategy within its feasible range for minimizing the total expected utilities of the workers throughout all sensing regions for each interval, without concerning about the actions a worker may take. Through turning the worker-customized strategy to an interval-customized one, the proposed combinatorial pinning ZD strategy reduces the number of the requestor's pricing strategies from $O\left(n^{3}\right)$ to $O(n)$. We evaluate the performance of the proposed cost-efficient MCS based on the check-in data from Foursquare. The simulation results verify that our scheme can always pin the expected utilities of the workers at a stably minimum level except for the situation with an irrational requestor adopting the ALLD strategy. Moreover, when the number of workers increases, the requestor can decrease her cooperation probability since the demand of high-quality sensing data from an individual worker decreases.

In our future research, we will consider the impact of mobility prediction on task assignments in MCS, which may result in more efficient MCS by computing mobile paths in advance; we also will investigate the challenges brought by the heterogeneity of the workers' various sensing abilities in MCS.

\section{ACKNOWLEDGMENT}

This work is partially supported by the US NSF under grants IIS-1741279 and CNS-1704397, and the National Natural Science Foundation of China under grant 61772080.

\section{REFERENCES}

[1] H. Jin, L. Su, D. Chen, H. Guo, K. Nahrstedt, and J. Xu, “Thanos: Incentive mechanism with quality awareness for mobile crowd sensing," IEEE Transactions on Mobile Computing, 2018.

[2] E. Wang, Y. Yang, J. Wu, W. Liu, and X. Wang, "An efficient prediction-based user recruitment for mobile crowdsensing," IEEE Transactions on Mobile Computing, vol. 17, no. 1, pp. 16-28, 2018.

[3] S. H. Marakkalage, S. Sarica, B. P. L. Lau, S. K. Viswanath, T. Balasubramaniam, C. Yuen, B. Yuen, J. Luo, and R. Nayak, "Understanding the lifestyle of older population: Mobile crowdsensing approach," IEEE Transactions on Computational Social Systems, vol. 6, no. 1, pp. 82-95, 2019.

[4] X. Wang, Y. Sui, J. Wang, C. Yuen, and W. Wu, "A distributed truthful auction mechanism for task allocation in mobile cloud computing," IEEE Transactions on Services Computing, 2018.

[5] Y. Chon, N. D. Lane, F. Li, H. Cha, and F. Zhao, "Automatically characterizing places with opportunistic crowdsensing using smartphones," in Proceedings of the 2012 ACM Conference on Ubiquitous Computing. ACM, 2012, pp. 481-490.

[6] R. K. Ganti, N. Pham, H. Ahmadi, S. Nangia, and T. F. Abdelzaher, "Greengps: a participatory sensing fuel-efficient maps application," in Proceedings of the 8th international conference on Mobile systems, applications, and services. ACM, 2010, pp. 151-164. 


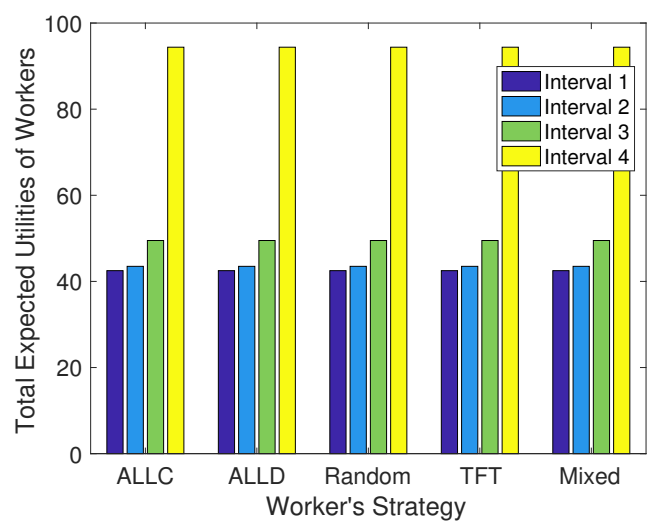

(a) CPZD

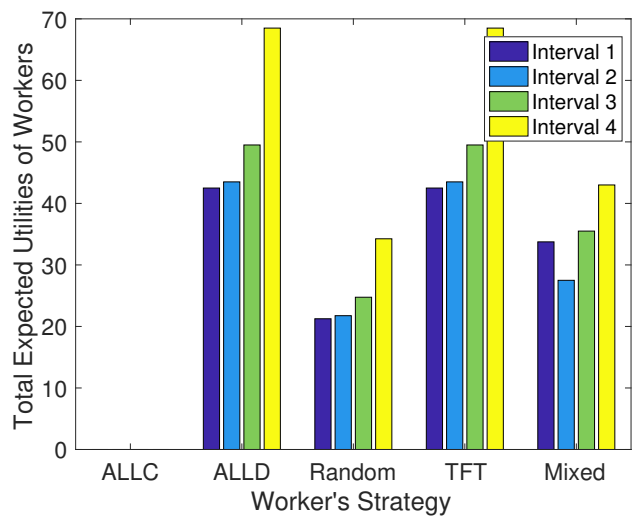

(c) ALLD

Fig. 7. Total expected utilities of the workers in all intervals.
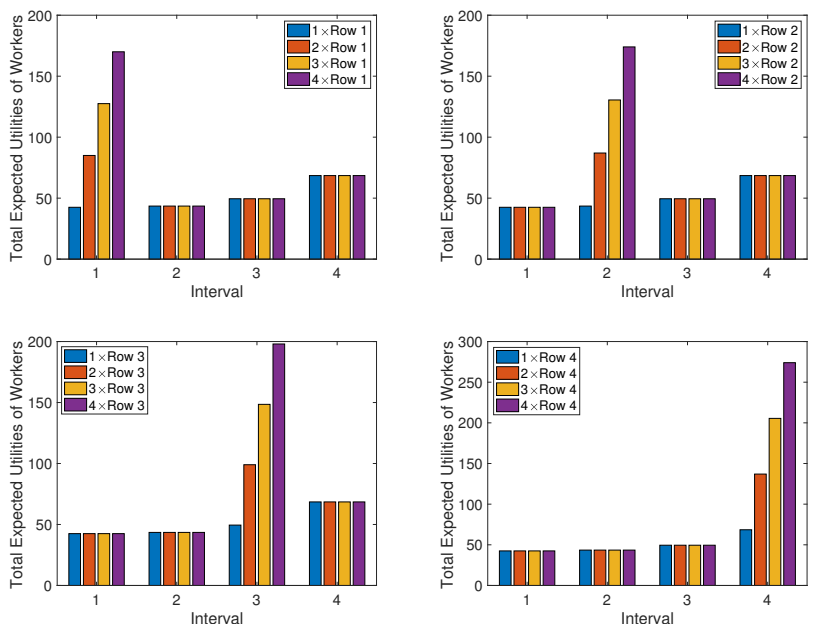

Fig. 8. Minimum total expected utilities in each interval when the row of A changes.

[7] J. Wang, N. Tan, J. Luo, and S. J. Pan, "Woloc: Wifi-only outdoor localization using crowdsensed hotspot labels," in INFOCOM 2017-IEEE Conference on Computer Communications. IEEE, 2017, pp. 1-9.

[8] Field agent. [Online]. Available: https://www.fieldagent.net/

[9] Gigwalkt. [Online]. Available: http://www.gigwalk.com/

[10] Waze. [Online]. Available: https://www.waze.com/zh/

[11] Million Agent.

[Online].

Available: http://www.millionagents.com/

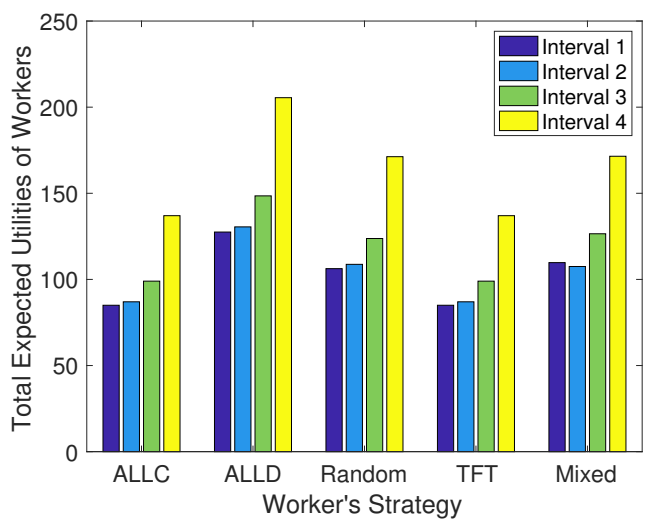

(b) ALLC

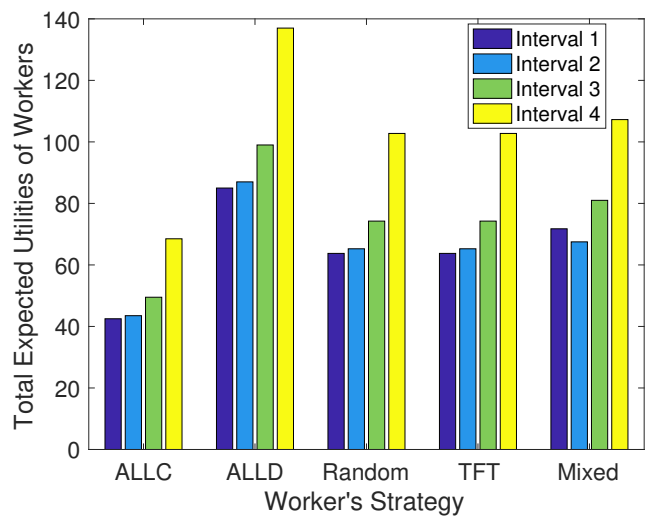

(d) Random
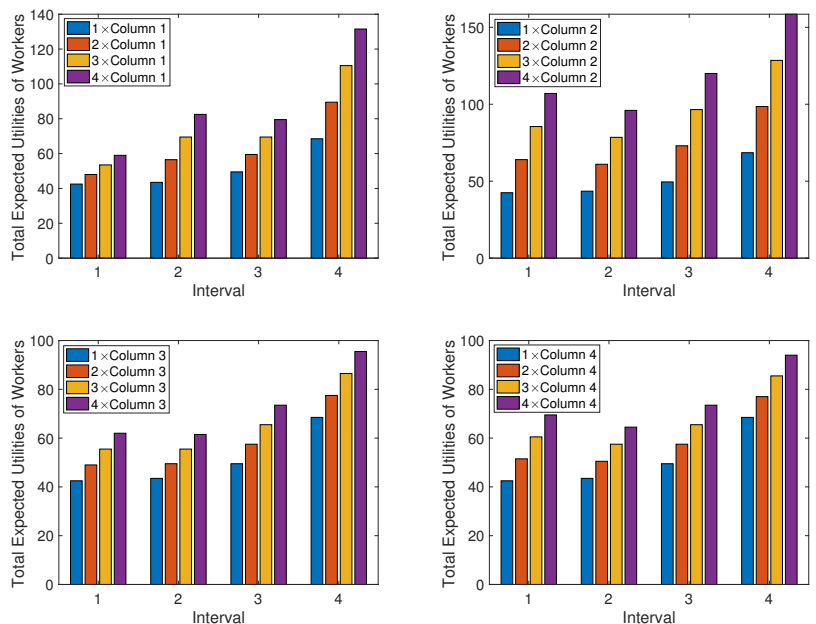

Fig. 9. Minimum total expected utilities in each interval when the column of $\mathbf{A}$ changes.

[12] D. Yang, D. Zhang, V. W. Zheng, and Z. Yu, "Modeling user activity preference by leveraging user spatial temporal characteristics in lbsns," IEEE Transactions on Systems, Man, and Cybernetics: Systems, vol. 45, no. 1, pp. 129-142, 2015.

[13] Z. Song, C. H. Liu, J. Wu, J. Ma, and W. Wang, "Qoi-aware multitask-oriented dynamic participant selection with budget constraints," IEEE Transactions on Vehicular Technology, vol. 63, no. 9, pp. 4618-4632, 2014.

[14] M. Zhang, P. Yang, C. Tian, S. Tang, X. Gao, B. Wang, and F. Xiao, 

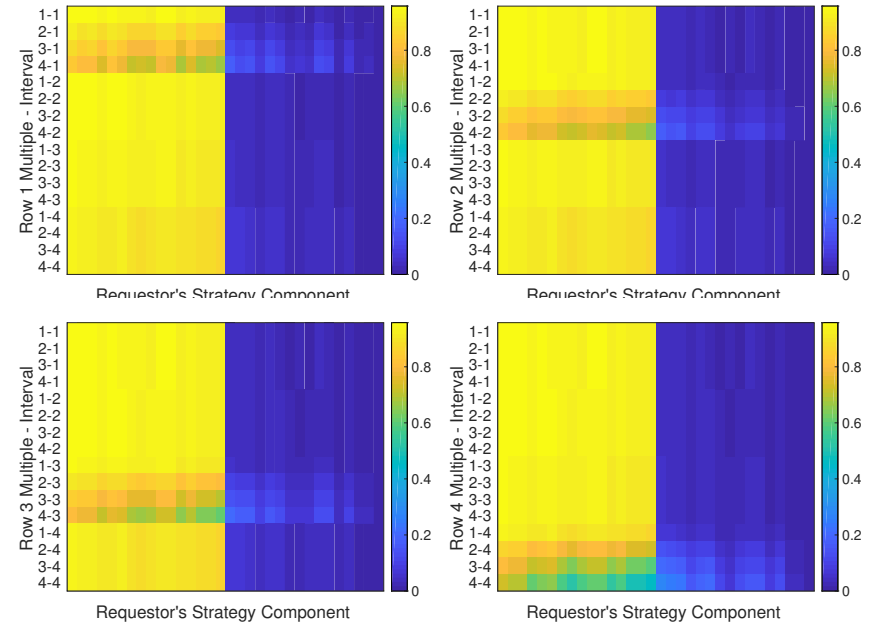

Fig. 10. Strategy of the requestor in each interval when the row of $\mathbf{A}$ changes.
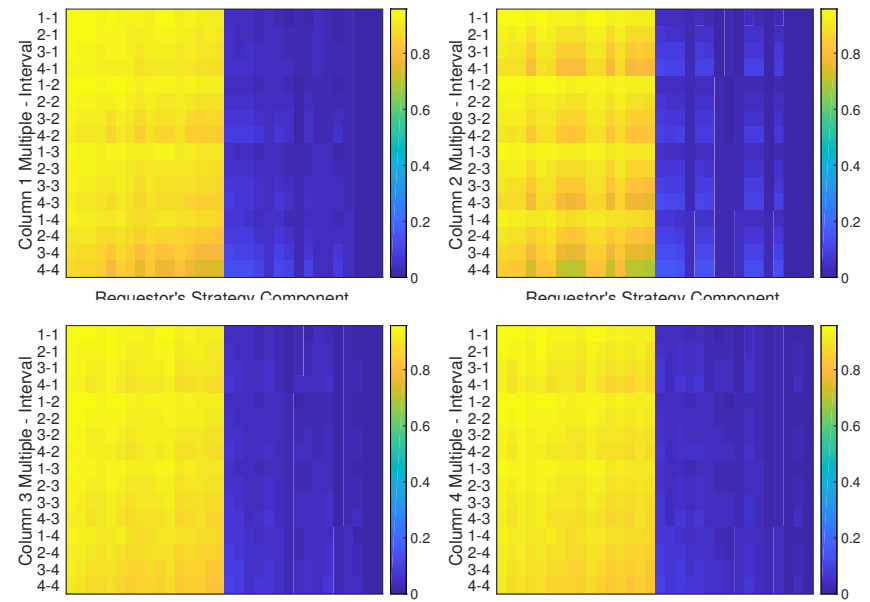

Requestor's Strategy Component

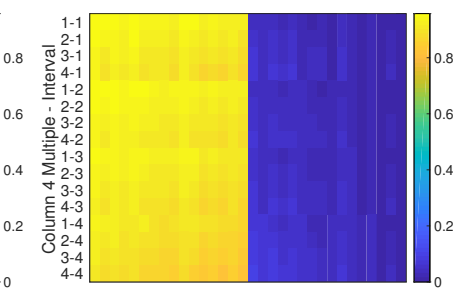

Requestor's Strategy Component

Fig. 11. Strategy of the requestor in each interval when the column of $\mathbf{A}$ changes.

"Quality-aware sensing coverage in budget-constrained mobile crowdsensing networks," IEEE Transactions on Vehicular Technology, vol. 65, no. 9, pp. 7698-7707, 2016.

[15] X. Zhang, Z. Yang, Y.-J. Gong, Y. Liu, and S. Tang, "Spatialrecruiter: maximizing sensing coverage in selecting workers for spatial crowdsourcing," IEEE Transactions on Vehicular Technology, vol. 66, no. 6, pp. 5229-5240, 2017.

[16] R. Estrada, R. Mizouni, H. Otrok, A. Ouali, and J. Bentahar, "A crowd-sensing framework for allocation of time-constrained and location-based tasks," IEEE Transactions on Services Computing, 2017.

[17] J. Wang, Y. Wang, D. Zhang, F. Wang, H. Xiong, C. Chen, Q. Lv, and Z. Qiu, "Multi-task allocation in mobile crowd sensing with individual task quality assurance," IEEE Transactions on Mobile Computing, 2018.

[18] L. Wang, D. Zhang, D. Yang, A. Pathak, C. Chen, X. Han, H. Xiong, and Y. Wang, "Space-ta: Cost-effective task allocation exploiting intradata and interdata correlations in sparse crowdsensing," ACM Transactions on Intelligent Systems and Technology (TIST), vol. 9, no. 2, pp. 20:1-20:28, 2017.

[19] Y. Wen, J. Shi, Q. Zhang, X. Tian, Z. Huang, H. Yu, Y. Cheng, and $X$. Shen, "Quality-driven auction-based incentive mechanism for mobile crowd sensing," IEEE Transactions on Vehicular Technology, vol. 64, no. 9, pp. 4203-4214, 2015.

[20] H. Jin, L. Su, D. Chen, K. Nahrstedt, and J. Xu, "Quality of information aware incentive mechanisms for mobile crowd sensing systems," in Proceedings of the 16th ACM International
Symposium on Mobile Ad Hoc Networking and Computing. ACM, 2015, pp. 167-176.

[21] D. Peng, F. Wu, and G. Chen, "Data quality guided incentive mechanism design for crowdsensing," IEEE Transactions on Mobile Computing, vol. 17, no. 2, pp. 307-319, 2018.

[22] W. H. Press and F. J. Dyson, "Iterated prisoner's dilemma contains strategies that dominate any evolutionary opponent," Proceedings of the National Academy of Sciences, vol. 109, no. 26, pp. 10409 $10413,2012$.

[23] K. Han, H. Huang, and J. Luo, "Quality-aware pricing for mobile crowdsensing," IEEE/ACM Transactions on Networking, vol. 99, no. 99, pp. 1-14, 2018.

[24] X. Tian, W. Zhang, Y. Yang, X. Wu, Y. Peng, and X. Wang, “Towards a quality-aware online pricing mechanism for crowdsensed wireless fingerprints," IEEE Transactions on Vehicular Technology, vol. 99, no. 99, pp. 1-12, 2018.

[25] L. Pan, D. Hao, Z. Rong, and T. Zhou, "Zero-determinant strategies in iterated public goods game," Scientific reports, vol. 5, p. 13096, 2015.

[26] C. Hilbe, B. Wu, A. Traulsen, and M. A. Nowak, "Cooperation and control in multiplayer social dilemmas," Proceedings of the National Academy of Sciences, vol. 111, no. 46, pp. 16425-16430, 2014.

[27] C. Hilbe, B. Wu, A. Traulsen, and M. A. Nowak, "Evolutionary performance of zero-determinant strategies in multiplayer games," Journal of theoretical biology, vol. 374, pp. 115-124, 2015.

[28] Q. Hu, S. Wang, P. Ma, X. Cheng, W. Lv, and R. Bie, "Quality control in crowdsourcing using sequential zero-determinant strategies," IEEE Transactions on Knowledge and Data Engineering, 2019.

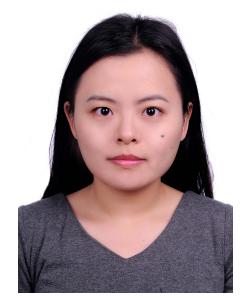

Qin Hu received her Ph.D. degree in Computer Science from the George Washington University in 2019. She is currently an Assistant Professor in the department of Computer and Information Science, Indiana University - Purdue University Indianapolis. Her research interests include wireless network, mobile security and privacy, crowdsourcing/crowdsensing and blockchain.

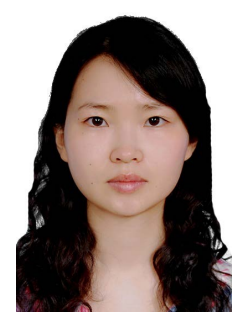

Shengling Wang is an associate professor in the College of Information Science and Technology, Beijing Normal University. She received her Ph.D. in 2008 from Xi'an Jiaotong University. After that, she did her postdoctoral research in the Department of Computer Science and Technology, Tsinghua University. Then she worked as an assistant and associate professor from 2010 to 2013 in the Institute of Computing Technology of the Chinese Academy of Sciences. Her research interests include mobile/wireless networks, game theory, crowdsourcing. 


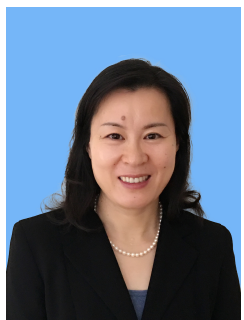

Xiuzhen Cheng $[F]$ received her M.S. and Ph.D. degrees in computer science from the University of Minnesota Twin Cities in 2000 and 2002, respectively. She is a professor in the Department of Computer Science, The George Washington University, Washington, DC. Her current research interests focus on privacyaware computing, wireless and mobile security, smart cities, and algorithm design and analysis. She has served on the Editorial Boards of several technical publications and the Technical Program Committees of various professional conferences/workshops. She has also chaired several international conferences. She worked as a program director for the U.S. National Science Foundation (NSF) from April to October 2006 (full time), and from April 2008 to May 2010 (part time).

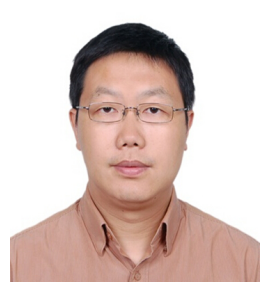

Weifeng Lv received his Ph.D. degree in computer science from Beihang University. His research interests include massive information system, urban cognitive computing, swarm intelligence, and smart cities. $\mathrm{He}$ is a professor of computer science, the dean of the School of Computer Science and Engineering, and the vice director of the State Key Laboratory of Software Development Environment, at Beihang University. He also serves as the Secretary-General of the China Software Industry Association and the director of National Engineering Research Center for Science and Technology Resources Sharing Service. He obtained multiple internationally renowned awards, including the second prize of the 2016 China National Science and Technology Invention Award and the first prize of the 2010 Beijing Science and Technology Award.

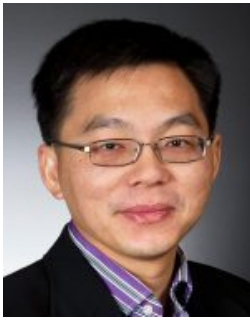

Junshan Zhang $[F]$ received the Ph.D. degree from the School of Electrical and Computer Engineering, Purdue University, West Lafayette, IN, USA, in 2000. In August 2000, he joined the School of Electrical, Computer, and Energy Engineering, Arizona State University, Tempe, AZ, USA, where he has been a Professor since 2010. His research interests fall in the general field of information networks and its intersections with power networks and social networks. His current research focuses on fundamental problems in information networks and energy networks, including modeling and optimization for smart grids, optimization/control of mobile social networks and cognitive radio networks, and privacy/security in information networks. Dr. Zhang received the Office of Naval Research Young Investigator Award in 2005 and the National Science Foundation CAREER Award in 2003. He also received the Outstanding Research Award from the IEEE Phoenix Section in 2003. He coauthored two papers that won him the Best Paper Runner-up Award at the 2009 IEEE International Conference on Computer Communications (INFOCOM) and the 2014 IEEE INFOCOM, as well as a paper that won him the Best Paper Award at the 2008 IEEE International Conference on Communications. He has been a Technical Program Committee (TPC) Cochair for a number of major conferences in communication networks, including the 2012 IEEE INFOCOM, the 2008 International Wireless Internet Conference, and the 2006 International Performance Computing and Communications Conference, as well as a TPC Vice Chair for the 2006 International Conference on Computer Communication and Networks. He was also the General Chair of the 2007 IEEE Communication Theory Workshop. He was an Associate Editor of the IEEE TRANSACTIONS ON WIRELESS COMMUNICATIONS and an Editor of Computer Networks and of the IEEE Wireless Communications Magazine. He was a Distinguished Lecturer of the IEEE Communications Society. He is currently serving as an Editor-at-Large for the IEEE/ACM TRANSACTIONS ON NETWORKING and an Editor for the IEEE Network Magazine. He is a TPC Cochair for the 2015 ACM International Symposium on Mobile Ad Hoc Networking and Computing. 